\title{
Antipsychotic polypharmacy in adult patients diagnosed with schizophrenia: A retrospective study
}

\author{
BOGDAN PATRICHI ${ }^{1,2}$, CRISTIANA ŢĂPOI ${ }^{1}$, RADU ŞTEFAN ROGOJINA $\breve{~}^{1}$, IRINA BEDREAGA $^{1}$, \\ ANCA DUMITRACHE $^{1}$, ANDREEA ITU ${ }^{1}$, RADU DRAGOMIR ${ }^{1}$ and ADELA-GEORGIANA BUCIUC ${ }^{1}$ \\ ${ }^{1}$ Department of General Psychiatry, 'Prof. Dr. Alexandru Obregia' Clinical Psychiatry Hospital, 041914 Bucharest;
${ }^{2}$ Department of General Psychiatry, 'Carol Davila' University of Medicine and Pharmacy, 050474 Bucharest, Romania
}

Received June 25, 2021; Accepted July 27, 2021

DOI: $10.3892 / \mathrm{etm} .2021 .10659$

\begin{abstract}
Antipsychotic polypharmacy (APP) is a common practice in the treatment of schizophrenia. In this study, we aimed to identify the prevalence of APP in our department, as well as the trends associated with co-prescribing antipsychotics. We collected data from the medical records of all 193 inpatients diagnosed with schizophrenia who were admitted to Prof. Dr. Alexandru Obregia Clinical Psychiatry Hospital (Bucharest, Romania), Department 9, during January 2019-December 2019. Demographic characteristics of the patients, clinical diagnosis, psychiatric admission type and duration of hospitalization were examined. Data regarding the antipsychotic regimen at discharge and other psychotropic drugs used were collected. A total of 69 (35.75\%) patients received more than 2 antipsychotics upon discharge. Patients treated with APP did not differ in regards to sex, age, education level, employment status, marital status, living situation, type of admission from those receiving antipsychotic monotherapy (APM). Prolonged hospitalization was found to be an independent predictor of APP $(\mathrm{P}=0.014)$. Most of the combinations used in our unit included clozapine $(47.8 \%)$, and the most frequently used treatment in the APP group was the combination of paliperidone and clozapine (14.5\%). In the APP group, $30(43.5 \%)$ patients included in their regimen was a long-acting intramuscular antipsychotic. There was no significant difference in terms of the use of mood stabilizers, antiparkinsonian drugs or anxiolytics between the APP and the APM group; yet, a higher prevalence of antidepressant use, although not statistically significant $(\mathrm{P}=0.067)$, in the APP group compared to the APM group, was observed. The use of APP as a long-term regimen is a common practice in our department, as it is worldwide. There is a great need for
\end{abstract}

Correspondence to: Dr Bogdan Patrichi, Department of General Psychiatry, 'Prof. Dr. Alexandru Obregia' Clinical Psychiatry Hospital, 10 Sos. Berceni, 041914 Bucharest, Romania

E-mail: bogdan.patrichi@umfcd.ro

Key words: schizophrenia, antipsychotic polypharmacy, co-prescribing, clozapine randomized-control trials and evidence-based studies in order to define the safest and most effective combinations of antipsychotics and also the characteristics of patients that may benefit from these combinations.

\section{Introduction}

Despite treatment guidelines advising against it, antipsychotic polypharmacy (APP), defined as the use of 2 or more antipsychotics simultaneously, is a common practice in the treatment of schizophrenia $(1,2)$.

Treatment resistance and low improvement of functional outcomes continue to be of significant concern among patients diagnosed with schizophrenia (3-5) and no antipsychotic has proved efficacious in treatment-resistant schizophrenia, with the exception of clozapine, which consistently results in significantly superior outcomes compared with other antipsychotics in patients unresponsive or partially responsive to antipsychotic monotherapies (1). Nonetheless, not all patients benefit from clozapine and not all of them tolerate it. In this context, APP is frequently used in clinical practice $(1,3)$.

Although treatment guidelines clearly state that patients with schizophrenia should be given a series of monotherapies, clozapine included, before trying APP, and that only approximately $15 \%$ of patients with schizophrenia (clozapine-resistant patients) should be administered APP (6-9), it is not always like this in clinical practice. In the last decade, many studies have noted an increase of APP in the treatment of schizophrenia; a recent meta-analysis reporting a prevalence of $10-57.7 \%$ (3).

Recently, the use of APP is of major interest in the scientific literature, with cross-sectional studies evaluating its use (10-12). There are several articles that have focused on the risks and benefits of APP and suggest that APP may prove advantageous in selected cases, but the studies cited are too heterogeneous to drive firm clinical recommendations (13-16). The combination aripiprazole-clozapine was found to be associated with the lowest risk of rehospitalization (13), which indicates that certain APP regimens may prove to have beneficial effects. Adding aripiprazole to clozapine may also prove useful as a weight reduction and lipid abnormality correction strategy $(11,17)$. While earlier studies have linked APP with increased mortality (18), recent studies have found no association between APP and mortality 
risk $(11,19,20)$. Still, APP remains controversial, with insufficient evidence supporting efficacy, long-term safety and concerns about increased costs.

The use of APP is common during acute exacerbation of symptoms in difficult-to-treat patients and during cross-titration from one antipsychotic to another, but APP is also used as a long-term strategy in treatment-resistant schizophrenia $(12,21-23)$. Many clinicians are reluctant to taper off one of the antipsychotic agents after the remission of an acute psychotic exacerbation, thus many patients remain 'stuck' with APP (24). On the long term, a complicated treatment regimen will lead to an accumulation of adverse reactions and it is more likely to be associated with poor treatment adherence. However, studies show that $50-67 \%$ of patients tolerate well when switching off polypharmacy and receiving antipsychotic monotherapy (APM) (25).

There is a discrepancy between recommendations from guidelines, the lack of high-quality evidence of APP efficacy and the real world clinical settings, where clinicians seem to be very fond of APP. The frequently mentioned reasons for APP include the inefficacy of a single antipsychotic, clozapine intolerance, the need for a rapid therapeutic response, severe course of illness, amelioration of side effects and skepticism about following treatment guidelines $(15,26-28)$. Other patient characteristics associated with the choice of APP are refractory-positive symptoms, uncontrolled impulsivity, violence, aggression, active substance abuse, severe affective symptoms, and borderline or antisocial personality traits $(24,29,30)$.

The aim of this study was to identify the prevalence of APP in our department, as well as the trends associated with co-prescribing antipsychotics and the characteristics of the patients who are most likely to receive APP.

\section{Patients and methods}

This study was designed as a retrospective review. We collected data from the medical records of all inpatients diagnosed with schizophrenia according to International Statistical Classification of Diseases and Related Health Problems, Tenth Revision (ICD-10) (31) who were admitted to Prof. Dr. Alexandru Obregia Clinical Psychiatry Hospital (Bucharest, Romania), Department 9, during January 2019-December 2019. For patients who had more than one admission during this period, we examined the record of the last hospitalization.

Demographic information of the patients, including sex, age, education, occupational status, marital status and living situation was examined. Clinical diagnosis, psychiatric admission type (voluntary/compulsory) and duration of hospitalization were examined. Data regarding the antipsychotic regimens tried during hospitalization and the treatment regimen at discharge, including forms of administration (oral, depot) and other psychotropic drug classes (antiparkinsonian drugs, antidepressants, anxiolytics and mood stabilizers) were collected.

Analyses were conducted using SPSS 27 package (IBM Corp.). Categorical variables were described in terms of proportions and continuous variables were described in terms of mean, standard deviation (SD), median and range. Chi-square test and Fisher's exact test were applied when comparing categorical variables. t-test and Wilcoxon test were applied for comparing means between groups. Significance was set at a P-value of 0.05 .

A multiple logistic regression was performed in order to identify the independent variables associated with APP. The logistic regression model included demographic features (sex, education, age, marital status, living situation, employment status, education level) and clinical features (type of admission, duration of hospitalization) as potential predictors for receiving APP.

\section{Results}

In 2019, 193 patients diagnosed with schizophrenia were admitted to our unit. Of these, 69 (35.75\%) received $>2$ antipsychotics upon discharge. We compared the group of patients who received one antipsychotic to the group of those patients treated with APP and noted that patients treated with APP did not differ in regards to sex, age, education level, employment status, marital status, living situation, type of admission form those receiving APM. Patients receiving APP tended to spend more days in the hospital (mean 19 days, standard deviation $\mathrm{SD}, 13$ ) compared to patients receiving APM (mean 13 days, $\mathrm{SD}, 10.6)$, and the difference was statistically significant $(\mathrm{P}=0.014)$.

Using multiple logistic regression, we identified that prolonged hospitalization was an independent predictor of APP [odds ratio (OR), 1.028, confidence interval (CI), 1.005-1.051, $\mathrm{P}=0.014]$. No significant predictive power was found for any socio-demographic characteristic or for the admission type. The characteristics of our sample are summarized in Table I.

Ten antipsychotics (amisulpride, aripiprazole, clozapine, flupenthixol decanoate, haloperidol, paliperidone, olanzapine, risperidone, quetiapine, zuclopenthixol depot) were administered both in the APM and in the APP group. A total of 37 of the 193 patients received clozapine. Of these, 33 (89.2\%) received another antipsychotic. Of the 65 patients discharged with risperidone, 22 (33.8\%) received another antipsychotic. Of the 39 patients discharged with aripiprazole, 21 (53.8\%) had this agent included in APP. Of the 30 patients discharged with paliperidone, $12(57.1 \%)$ received it in combination with antipsychotics. Of the 31 patients discharged with quetiapine, $19(61.3 \%)$ received another antipsychotic. Of the 14 patients discharged with amisulpride, $11(78.6 \%)$ had an APP regimen.

Of the 69 patients who received APP, $2(2.9 \%)$ were treated with a combination of 3 antipsychotics, while67 (97.1\%) were treated with 2 antipsychotics. Most of the combinations used included 2 second-generation antipsychotics (SGAs) (81\%), followed by an SGA plus a first-generation antipsychotic (FGA) $(15 \%)$. Three (4\%) patients received a combination of 2 FGAs. In 11 of 13 cases (84.6\%) when patients received at least one FGA, this was administered as a long-acting injection.

Most of the combinations used in our unit included clozapine $(47.8 \%)$, followed by risperidone $(31.8 \%)$ and aripiprazole $(30.4 \%)$. The most frequently used treatment in the APP group was the combination of paliperidone and clozapine (14.5\%); the second and the third most used regimens also included clozapine combined with risperidone (13\%) and aripiprazole (11.6\%), respectively. Other frequently used 
Table I. Socio-demographic characteristics of the study sample of patients with schizophrenia $(\mathrm{N}=193)$.

\begin{tabular}{|c|c|c|c|}
\hline Variable & Single antipsychotic & Antipsychotic polypharmacy & P-value \\
\hline Sex, n (\%) & & & 0.889 \\
\hline Male & $57(46 \%)$ & $31(45 \%)$ & \\
\hline Female & $67(54 \%)$ & $38(55 \%)$ & \\
\hline Mean age (years) & 45 (SD 12) & $43(\mathrm{SD} 11)$ & 0.241 \\
\hline Marital status, n (\%) & & & 0.921 \\
\hline Single & $70(56.4 \%)$ & $40(58 \%)$ & \\
\hline Married/Partner & $27(21.8 \%)$ & $14(20.3 \%)$ & \\
\hline Divorced & $22(17.7 \%)$ & $13(18.9 \%)$ & \\
\hline Widowed & $2(1.6 \%)$ & $0(0 \%)$ & \\
\hline Living situation, $\mathrm{n}(\%)$ & & & 0.906 \\
\hline Alone & $23(18.6 \%)$ & $13(18.9 \%)$ & \\
\hline With spouse/partner & $27(21.8 \%)$ & $14(20.2 \%)$ & \\
\hline With parents/other relatives & $68(54.8 \%)$ & $38(55.1 \%)$ & \\
\hline Institutionalized & $5(4 \%)$ & $1(1.4 \%)$ & \\
\hline Employment, n (\%) & & & 0.918 \\
\hline Employed & $7(5.7 \%)$ & $5(7.3 \%)$ & \\
\hline Unemployed & $17(13.7 \%)$ & $9(13 \%)$ & \\
\hline Retired & $100(80.6 \%)$ & $55(79.7 \%)$ & \\
\hline Education level, n (\%) & & & 0.520 \\
\hline$\leq 8$ years & $16(13 \%)$ & $6(8.7 \%)$ & \\
\hline $8-12$ years & $50(40.3 \%)$ & $25(36.2 \%)$ & \\
\hline$>12$ years & $24(19.3 \%)$ & $17(24.6 \%)$ & \\
\hline Type of admission, n (\%) & & & 0.613 \\
\hline Voluntary & $80(64.5 \%)$ & $47(68.1 \%)$ & \\
\hline Compulsory & $44(35.5 \%)$ & $22(31.9 \%)$ & \\
\hline Duration of hospitalization (days) & $13(\mathrm{SD} 10.6)$ & $19(\mathrm{SD} 13)$ & 0.014 \\
\hline
\end{tabular}

Table II. Distribution of additional psychotropic drugs at discharge.

\begin{tabular}{lccr}
\hline Additional psychotropic drug & Single antipsychotic & Antipsychotic polypharmacy & $\chi^{2}, \mathrm{P}-\mathrm{value}$ \\
\hline Anticholinergic, n (\%) & $54(43.5)$ & $31(44.9)$ & $0.034, \mathrm{P}=0.853$ \\
Mood stabilizer, n (\%) & $69(55.6)$ & $36(52.2)$ & $0.215, \mathrm{P}=0.643$ \\
Benzodiazepine, n (\%) & $75(60.5)$ & $41(59.4)$ & $0.021, \mathrm{P}=0.885$ \\
Antidepressant, n (\%) & $28(22.6)$ & $24(34.8)$ & $3.353, \mathrm{P}=0.067$ \\
\hline
\end{tabular}

combinations were aripiprazole plus quetiapine (10.1\%) and risperidone plus quetiapine (7.2\%). The mean dose of clozapine administered at discharge in the APP group was $180 \mathrm{mg} /$ day, with a highest dose of $500 \mathrm{mg} /$ day and the lowest dose of $25 \mathrm{mg}$ /day. Most of the patients with APP that included clozapine received a dose of $100 \mathrm{mg} /$ day (42.4\%).

In the APP group, $30(43.5 \%)$ patients had in their regimen a long-acting intramuscular antipsychotic. The most frequently used long-acting antipsychotic was flupenthixol decanoate (30\%), followed by paliperidone depot (26.7\%) and risperidone depot (20\%). The most frequently occurring combinations in the long-acting group were paliperidone palmitate and clozapine (20\%), risperidone depot and clozapine (10\%), aripiprazole depot and clozapine (10\%) and flupenthixol decanoate and haloperidol (10\%). In the total sample, 62 of the patients received a long-acting antipsychotic, which means that almost half of them $(48.4 \%)$ had it associated with another antipsychotic.

In the APP group, 31 patients (44.9\%) received antiparkinsonian drugs, $36(52.2 \%)$ received mood stabilizers, $41(59.4 \%)$ received benzodiazepines. There was no significant difference in terms of the use of mood stabilizers, antiparkinsonian drugs or anxiolytics between the APP and the APM group. We observed a higher prevalence of antidepressant use in the APP group (34.8\% compared to $22.6 \%$ in the APM group), but this difference was not statistically significant $(\mathrm{P}=0.067)$. 
Information about the distribution of additional psychotropic medication used at discharge can be found in Table II.

\section{Discussion}

Approximately one third (35.7\%) of our patients received more than one antipsychotic at discharge. None of these patients was in the process of cross-titration, which suggests that the combination of antipsychotics was intended for long-term use. This finding is similar to the prevalence reported in other recent studies by Jaffe and Levine (31.1\%) (32), Lung et al (24.2\%) (10) and Suokas et al (46.2\%) (11).

There is no socio-demographic factor associated with a higher rate of APP, a finding supported by other studies $(12,33)$. Some studies observed a higher rate of APP administration in male patients rather than female patients, but this difference was not statistically significant $(10,33)$. Jaffe and Levine (32) found that patients over 65 years of age were less likely than younger patients to receive APP. The reason our data did not support this finding may be due to the fact that the oldest patient in our sample was 73 , and only 8 patients were older than 65 years.

A prolonged duration of hospitalization was a significant predictor $(\mathrm{P}=0.014)$ of the administration of APP. Similar findings were observed by Suokas et al (11), suggesting that patients who have an extended hospitalization period have a partial or unsatisfactory response to various treatment regimens. Lerma-Carrillo et al (33) observed no difference in the time of hospitalization between patients on APM or APP, but the mean time of hospitalization was higher in their sample (25.7 and 23.8 days for the APM and APP group, respectively).

Most of the combinations used in our sample included 2 SGAs (81\%), which is in accordance with other recent studies (10), suggesting that first-generation antipsychotics (FGAs) are used only in selected cases.

The most frequently used antipsychotics in the APP was clozapine. Moreover, $33(89 \%)$ of the patients who received clozapine in our sample also received another antipsychotic. This finding is particular, because in most of the studies concerning APP, clozapine was the antipsychotic least likely to be prescribed in combination $(32,34)$.

The most frequently used combinations were clozapine plus paliperidone, clozapine plus risperidone and clozapine plus aripiprazole. The fact that most APP regimens received by patients in our sample included clozapine could indicate that APP was used in treatment refractory cases or in cases where higher doses of clozapine were not well tolerated. However, the mean dose of clozapine was $180 \mathrm{mg} /$ day, which was significantly lower than the mean dose reported by other studies, where the clinicians reported doses from $492 \mathrm{mg} /$ day (35) to $557 \mathrm{mg} /$ day (33). This suggests that in our sample clozapine was used as an augmentation for other antipsychotics, rather than vice versa.

The combinations that include clozapine are the ones that were studied the most. Nonetheless, the data are rather inconclusive. The most frequent combination tested in randomized controlled trials was clozapine and risperidone (36), but few randomized controlled studies concerning the combination of risperidone and clozapine showed favorable results in certain situations (37-39). With regard to the clozapine plus aripiprazole combination, two studies $(40,41)$ showed a favorable metabolic impact when adding aripiprazole to clozapine, but no impact on the reduction of positive and negative symptoms. In our study, the reason why the combination clozapine plus aripiprazole was used was not determined.

Almost half of the patients in the APP group (43.5\%) received a long-acting antipsychotic, while almost half of the patients in our sample that received a long-acting antipsychotic (48.4\%) were in the APP group. The fact that a long-acting antipsychotic is used widely in APP regimens may suggest that treatment-resistant cases are associated with reduced medical adherence.

Our data showed that whenever a FGA was administered, this tended to be in a long-acting form (84.6\%). The analysis performed by Lung et al (10) revealed that, when using SGA in APP, these were more likely to be administered in oral form $(92.2 \%)$, while half of FGA were prescribed as depot injection.

In our sample, the group of APM did not differ from APP when assessed for the use of other psychotropic agents. This is in contrast to the findings of other investigators, who showed that clinicians are less likely to prescribe mood stabilizers or benzodiazepines when treating patients with APP (33) and more likely to prescribe antiparkinsonian drugs for patients treated with APP (12). The use of antiparkinsonian drugs in our sample was $44 \%$, which is similar to the findings of Procyshyn et al (34) who reported a $42 \%$ use of antiparkinsonian drugs associated with antipsychotic treatment.

Although not statistically significant, we observed that patients receiving APP had a higher prevalence of antidepressants. This result was not in line with other studies $(11,16)$, which reported a lower use of antidepressants among patients treated with APP. All of these differences may be explained by both the heterogeneity of the samples and by the individual differences in clinical manifestations of schizophrenia.

In conclusion, the use of APP as a long-term regimen is a common practice in our department, as it is worldwide. Almost half of the antipsychotic combinations included clozapine and most of these combinations also included a long-acting antipsychotic. The length of hospital stay was an important predictor for the recommendation of a combination of antipsychotics at discharge. There is a great need of randomized-control trials and evidence-based studies in order to define the safest and most effective combinations of antipsychotics and also the characteristics of patients that may benefit from these combinations.

\section{Acknowledgements}

Not applicable.

\section{Funding}

No funding was received.

\section{Availability of data and materials}

The datasets used during the current study are available from the corresponding author on request. 


\section{Authors' contributions}

CT, AGB, RȘR, IB, AD, AI, and RD contributed to the data acquisition. BP contributed to the conception of the study and the revision of the manuscript. CT contributed to the design and conception of the study, the analysis and the interpretation of data. BP and CT confirmed the authenticity of the raw data. All authors read and approved the final manuscript for publication.

\section{Ethics approval and consent to participate}

Approval was obtained from the Ethics Committee of the 'Prof. Dr. Alexandru Obregia' Clinical Psychiatry Hospital, Bucharest, Romania (approval no. 67/2021). All patients signed the informed consent form for research.

\section{Patients consent for publication}

Not applicable.

\section{Competing interests}

The authors declare that they have no competing interests.

\section{References}

1. National Collaborating Centre for Mental Health: Schizophrenia: The NICE Guideline on Core Interventions in the Treatment and Management of Schizophrenia in Adults in Primary and Secondary Care (updated edition). The British Psychological Society and the Royal College of Psychiatrists. London, 2010.

2. Moore TA, Buchanan RW, Buckley PF, Chiles JA, Conley RR, Crismon ML, Essock SM, Finnerty M, Marder SR, Miller DD, et al: The Texas Medication Algorithm Project antipsychotic algorithm for schizophrenia: 2006 update. J Clin Psychiatry 68: 1751-62, 2007.

3. Correll C, Rummel-Kluge C, Corves C, Kane J and Leucht S: Antipsychotic combinations vs. monotherapy in schizophrenia: A meta-analysis of randomized controlled trials. Schizophr Bull 35: 443-457, 2009.

4. Jones PB, Barnes TR, Davies L, Dunn G, Lloyd H, Hayhurst K, Murray R, Markwick A and Lewis S: Randomized controlled trial of the effect on Quality of Life of second- vs. first generation antipsychotic drugs in schizophrenia: Cost Utility of the Latest Antipsychotic Drugs in Schizophrenia Study (CUtLASS 1). Arch Gen Psychiatry 63: 1079-1087, 2006.

5. Lieberman JA, Stroup TS, McEvoy JP, Swartz M, Rosenheck R, Perkins D, Keefe R, Davis S, Davis C, Lebowitz B, et al: Effectiveness of antipsychotic drugs in patients with chronic schizophrenia. N Engl J Med 353: 1209-1223, 2005.

6. Baandrup L, Allerup P, Lublin H, Nordentoft M, Peacock L and Glenthoj B: Evaluation of a multifaceted intervention to limit excessive antipsychotic co-prescribing in schizophrenia out-patients. Acta Psychiatr Scand 122: 367-374, 2010

7. Clark RE, Bartels SJ, Mellman TA and Peacock WJ: Recent trends in antipsychotic combination therapy of schizophrenia and schizoaffective disorder: Implications for state mental health policy. Schizophr Bull 28: 75-84, 2002.

8. Ganguly R, Kotzan JA, Miller LS, Kennedy K and Martin BC: Prevalence, trends, and factors associated with antipsychotic polypharmacy among Medicaid-eligible schizophrenia patients, 1998-2000. J Clin Psychiatry 65: 1377-1388, 2004

9. Paton C, Lelliott P, Harrington M, Okocha C, Sensky T and Duffett R: Patterns of antipsychotic and anticholinergic prescribing for hospital inpatients. J Psychopharmacol 17: 223-229, 2003.

10. Lung SLM, Lee HME, Chen YHE, Chan KWS, Chang WC and Hui LMC: Prevalence and correlates of antipsychotic polypharmacy in Hong Kong. Asian J Psychiatr 33: 113-120, 2018.
11. Suokas J, Suvisaari J, Haukka J, Korhonen P and Tiihonen J: Description of long-term polypharmacy among schizophrenia outpatients. Soc Psychiatry Psychiatr Epidemiol 48: 631-638, 2013.

12. Santone G, Bellantuono C, Rucci P, Picardi A, Preti A and de Girolamo G: Patient characteristics and process factors associated with antipsychotic polypharmacy in a nationwide sample of psychiatric inpatients in Italy. Pharmacoepidemiol Drug Saf 20: 441-449, 2011.

13. Tiihonen J, Taipale H, Mehtälä J, Vattulainen P, Correll C and Tanskanen A: Association of antipsychotic polypharmacy vs. monotherapy with psychiatric rehospitalization among adults with schizophrenia. JAMA Psychiatry 76: 499-507, 2019.

14. Fleischhacker $\mathrm{W}$ and Uchida $\mathrm{H}$ : Critical review of antipsychotic polypharmacy in the treatment of schizophrenia. Int J Neuropsychopharmacol 17: 1083-1093, 2014.

15. Lin SK: Antipsychotic polypharmacy: A dirty little secret or a fashion? Int J Neuropsychopharmacol 23: 125-131, 2020.

16. Gallego J, Bonetti J, Zhang J, Kane J and Correll C: Prevalence and correlates of antipsychotic polypharmacy: A systematic review and meta-regression of global and regional trends from the 1970s to 2009. Schizophr Res 138: 18-28, 2012.

17. Trifu S, Vlăduţi A and Trifu AI: Genetic aspects in schizophrenia. Receptoral theories. Metabolic theories. Rom J Morphol Embryol 61: 25-32, 2020.

18. Joukamaa M, Heliövaara M, Knekt P, Aromaa A, Raitasalo R and Lehtinen V: Schizophrenia, neuroleptic medication and mortality. Br J Psychiatry 188: 122-127, 2006.

19. Tiihonen J, Suokas J, Suvisaari J, Haukka J and Korhonen P: Polypharmacy with antipsychotics, antidepressants, or benzodiazepines and mortality in schizophrenia. Arch Gen Psychiatry 69: 476-483, 2012.

20. Baandrup L, Gasse C, Jensen V, Glenthoj B, Nordentoft M, Lublin H, Fink-Jensen A, Lindhart A and Mortensen P: Antipsychotic polypharmacy and risk of death from natural causes in patients with schizophrenia: A population-based nested case-control study. J Clin Psychiatry 71: 103-108, 2010.

21. Mauri MC, Regispani F, Beraldo S, Volonteri L, Ferrari V, Fiorentini A and Invernizzi G: Patterns of clinical use of antipsychotics in hospitalized psychiatric patients. Prog Neuropsychopharmacol Biol Psychiatry 29: 957-963, 2005.

22. Sim K, Su A, Fujii S, Yang SY, Chong MY, Ungvari G, Si T, Chung EK, Tsang HY, Chan YH, et al: Antipsychotic polypharmacy in patients with schizophrenia: A multicentre comparative study. Br J Clin Pharmacol 58: 178-183, 2004.

23. Morrato EH, Dodd S, Oderda G, Haxby DG, Allen R and Valuck RJ: Prevalence, utilization patterns, and predictors of antipsychotic polypharmacy: Experience in a multisite Medicaid population, 1998-2003. Clin Ther 29: 183-195, 2007.

24. Tapp A, Ernst Wood A, Secrest L, Erdmann J, Cubberley L and Kilzieh N: Combination antipsychotic therapy in clinical practice. Psychiatr Serv 54: 55-59, 2003.

25. Suzuki T, Uchida H, Watanabe K, Yagi G and Kashima H: A clinical case series of switching from antipsychotic polypharmacy to monotherapy with a second generation agent on patients with chronic schizophrenia. Prog Neuropsychopharmacol Biol Psychiatry 28: 361-369, 2004.

26. Malandain L, Thibaut F, Grimaldi-Bensouda L, Falissard B Abenhaim L and Nordon C: Correlates and predictors of antipsychotic drug polypharmacy in real-life settings: Results from a nationwide cohort study. Schizophr Res 192: 213-218, 2018.

27. Ito H, Koyama A and Higuchi T: Polypharmacy and excessive dosing: Psychiatrists' perceptions of antipsychotic drug prescription. Br J Psychiatry 187: 243-247, 2005.

28. Barnes TR and Paton C: Antipsychotic polypharmacy in schizophrenia: Benefits and risks. CNS Drugs 25: 383 399, 2011.

29. Stahl S: Emerging guidelines for the use of antipsychotic polypharmacy. Rev Psiquiatr Salud Ment 6: 97-100, 2013 (In English, Spanish).

30. Trifu S, Tudor A and Rădulescu I: Aggressive behavior in psychiatric patients in relation to hormonal imbalance (Review). Exp Ther Med 20: 3483-3487, 2020.

31. World Health Organization (WHO): The ICD-10 Classification of Mental and Behavioural Disorders. Genève, Switzerland: World Health Organization, 1993. https://apps.who.int/iris/ handle $/ 10665 / 37108$.

32. Jaffe A and Levine J: Antipsychotic medication coprescribing in a large state hospital system. Pharmacoepidemiol Drug Saf 12: 41-48, 2003. 
33. Lerma-Carrillo I, de Pablo Brühlmann S, del Pozo ML, Pascual-Pinazo F, Molina J and Barca-García E: Antipsychotic Polypharmacy in Patients With Schizophrenia in a Brief Hospitalization Unit. Clin Neuropharmacol 31: 319-332, 2008.

34. Procyshyn RM, Kennedy NB, Tse G and Thompson B: Antipsychotic polypharmacy: A survey of discharge prescriptions from a tertiary care psychiatric institution. Can J Psychiatry 46: 334-339, 2001.

35. Honer WG, Thornton AE, Chen EY, Chan RC, Wong JO, Bergmann A, Falkai P, Pomarol-Clotet E, McKenna PJ, Stip E, et al: Clozapine alone versus clozapine and risperidone with refractory schizophrenia. N Engl J Med 354: 472-482, 2006.

36. Pandurangi A and Dalkilic A: Polypharmacy with second-generation antipsychotics: A review of evidence. J Psychiatr Pract 14: $345-367,2008$

37. Zink M, Kuwilsky A, Krumm B and Dressing H: Efficacy and tolerability of ziprasidone vs. risperidone as augmentation in patients partially responsive to clozapine: A randomised controlled clinical trial. J Psychopharmacol 23: 305-314, 2009.

38. Josiassen RC, Joseph A, Kohegyi E, Stokes S, Dadvand M, Paing WW and Shaughnessy R: Clozapine augmented with risperidone in the treatment of schizophrenia: A randomized, double-blind, placebo-controlled trial. Am J Psychiatry 162: 130-136, 2005.
39. Freudenreich O, Henderson DC, Walsh JP, Culhane MA and Goff D: Risperidone augmentation for schizophrenia partially responsive to clozapine: A double-blind, placebo-controlled trial. Schizophr Res 92: 90-94, 2007.

40. Stroup TS, McEvoy JP, Ring KD, Hamer RH, LaVange L, Swartz M, Rosenheck R, Perkins D, Nussbaum A and Lieberman JA; Schizophrenia Trials Network: A randomized trial examining the effectiveness of switching from olanzapine, quetiapine, or risperidone to aripiprazole to reduce metabolic risk: Comparison of antipsychotics for metabolic problems (CAMP). Am J Psychiatry 168: 947-956, 2011.

41. Fleischhacker WW, Heikkinen ME, Olie JP, Landsberg W, Dewaele P, McQuade R, Loze JY, Hennicken D and Kerselaers W: Effects of adjunctive treatment with aripiprazole on body weight and clinical efficacy in schizophrenia patients treated with clozapine: A randomized, double-blind, placebo-controlled trial. Int J Neuropsychopharmacol 13: 1115-1125, 2010.

This work is licensed under a Creative Commons Attribution-NonCommercial-NoDerivatives 4.0 International (CC BY-NC-ND 4.0) License. 